\title{
Limits on additional planetary companions to OGLE 2005-BLG-390L
}

D. Kubas ${ }^{1}$, A. Cassan ${ }^{2}$, M. Dominik ${ }^{3, \star}$, D. P. Bennett ${ }^{4}$, J. Wambsganss ${ }^{2}$, S. Brillant ${ }^{1}$, J. P. Beaulieu ${ }^{5}$, M. D. Albrow ${ }^{12}$, V. Batista ${ }^{5}$, M. Bode ${ }^{19}$, D. M. Bramich ${ }^{14}$, M. Burgdorf ${ }^{19}$, J. A. R. Caldwell ${ }^{18}$, H. Calitz $^{16}$, K. H. Cook ${ }^{21}$, Ch. Coutures ${ }^{8}$,

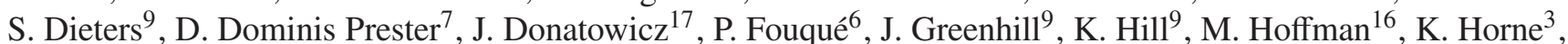
U. G. Jørgensen ${ }^{13}$, N. Kains ${ }^{3}$, S. Kane ${ }^{20}$, J. B. Marquette ${ }^{4}$, R. Martin ${ }^{11}$, P. Meintjes ${ }^{16}$, J. Menzies ${ }^{10}$, K. R. Pollard ${ }^{11}$, K. C. Sahu ${ }^{15}$, C. Snodgrass ${ }^{1}$, I. Steele ${ }^{19}$, Y. Tsapras ${ }^{22}$, C. Vinter ${ }^{13}$, A. Williams ${ }^{11}$, K. Woller ${ }^{13}$, and M. Zub ${ }^{2}$ (The PLANET/RoboNet Collaboration)

1 European Southern Observatory, Casilla 19001, Vitacura 19, Santiago, Chile e-mail: dkubas@eso.org

2 Astronomisches Rechen-Institut, Zentrum für Astronomie, Heidelberg University, Mönchhofstr. 12-14, 69120 Heidelberg, Germany

3 SUPA, University of St Andrews, School of Physics \& Astronomy, North Haugh, St Andrews, KY16 9SS, UK

${ }^{4}$ University of Notre Dame, Department of Physics, 225 Nieuwland Science Hall Notre Dame, USA

5 Institut d'Astrophysique de Paris, 98bis boulevard Arago, 75014 Paris, France

6 Observatoire Midi-Pyrénées, UMR 5572, 14 avenue Edouard Belin, 31400 Toulouse, France

7 Physics department, Faculty of Arts and Sciences, University of Rijeka, 51000 Rijeka, Croatia

DSM/DAPNIA, CEA Saclay, 91191 Gif-sur-Yvette Cedex, France

9 University of Tasmania, School of Maths and Physics, Private bag 37, GPO Hobart, Tasmania 7001, Australia

10 South African Astronomical Observatory, PO Box 9 Observatory 7935, South Africa

11 Perth Observatory, Walnut Road, Bickley, Perth 6076, Australia

12 University of Canterbury, Department of Physics \& Astronomy, Private Bag 4800, Christchurch, New Zealand

13 Niels Bohr Institute, Astronomical Observatory, Juliane Maries Vej 30, 2100 Copenhagen, Denmark

14 Isaac Newton Group, Apartado de Correos 321, 38700 Santa Cruz de La Palma, Spain

15 Space Telescope Science Institute, 3700 San Martin Drive, Baltimore, MD 21218, USA

16 Dept. of Physics / Boyden Observatory, University of the Free State, Bloemfontein 9300, South Africa

17 Technical University of Vienna, Dept. of Computing, Wiedner Hauptstrasse 10, Vienna, Austria

${ }_{18}$ McDonald Observatory, 16120 St Hwy Spur 78, Fort Davis, TX 79734, USA

19 Astrophysics Research Institute, Liverpool John Moores University, Twelve Quays House, Egerton Wharf, Birkenhead CH41 1LD, UK

20 Department of Astronomy, University of Florida, 211 Bryant Space Science Center, Gainesville, FL 32611-2055, USA

${ }^{21}$ Lawrence Livermore National Laboratory, IGPP, PO Box 808, Livermore, CA 94551, USA

22 Astronomy Unit, School of Mathematical Sciences, Queen Mary, University of London, Mile End Road, London E1 4NS, UK Received 9 March 2007 / Accepted 18 February 2008

\section{ABSTRACT}

\begin{abstract}
Aims. We investigate constraints on additional planets orbiting the distant M-dwarf star OGLE 2005-BLG-390L, around which photometric microlensing data has revealed the existence of the sub-Neptune-mass planet OGLE 2005-BLG-390Lb. We specifically aim to study potential Jovian companions and compare our findings with predictions from core-accretion and disc-instability models of planet formation. We also obtain an estimate of the detection probability for sub-Neptune mass planets similar to OGLE 2005-BLG390Lb using a simplified simulation of a microlensing experiment.

Methods. We compute the efficiency of our photometric data for detecting additional planets around OGLE 2005-BLG-390L, as a function of the microlensing model parameters and convert it into a function of the orbital axis and planet mass by means of an adopted model of the Milky Way.

Results. We find that more than 50\% of potential planets with a mass in excess of $1 M_{\mathrm{J}}$ between 1.1 and 2.3 AU around OGLE 2005BLG-390L would have revealed their existence, whereas for gas giants above $3 M_{\mathrm{J}}$ in orbits between 1.5 and $2.2 \mathrm{AU}$, the detection efficiency reaches 70\%; however, no such companion was observed. Our photometric microlensing data therefore do not contradict the existence of gas giant planets at any separation orbiting OGLE 2005-BLG-390L. Furthermore we find a detection probability for an OGLE 2005-BLG-390Lb-like planet of around 2-5\%. In agreement with current planet formation theories, this quantitatively supports the prediction that sub-Neptune mass planets are common around low-mass stars.
\end{abstract}

Key words. stars: planetary systems - gravitational lensing

\section{Introduction}

After more than a decade since the discovery of the first extrasolar planet orbiting a solar-type star (Mayor \& Queloz 1995)

^ Royal Society University Research Fellow. the Extra-solar Planet Encyclopedia ${ }^{1}$ lists 249 entries, including an increasing number (26) of multiple planetary systems. The accessible mass regime of extra-solar planets extends

\footnotetext{
1 http://exoplanet.eu
} 
since 2005 below the $10 M_{\oplus}$ regime, with the discoveries of Gliese $876 \mathrm{~d}\left(\sim 7.5 M_{\oplus}\right.$, Rivera et al. 2005) and the pair of planets around Gliese 581 with minimum masses of 5 and $8 M_{\oplus}$ (Udry et al. 2007) using the radial-velocity technique, as well as OGLE 2005-BLG-390Lb detected by microlensing ( $5 M_{\oplus}$, Beaulieu et al. 2006). While recent improvements in radial velocity sensitivity have enabled the discovery of Neptune-mass planets in Venus-like orbits (Lovis et al. 2006; Alibert et al. 2006), microlensing is the only method that can detect such sub-Neptune-mass planets in orbits beyond 1 AU. This sensitivity to sub-Neptune-mass planets at separations of a few AU is important for testing the core accretion theory of planet formation because this theory predicts that the dominant planets in any planetary system should form in the vicinity of the "snow line," which is located at a few AU (Kennedy et al. 2006; Ida \& Lin 2004; Laughlin et al. 2004). Microlensing results allow this theory to be tested without confronting the additional uncertainties of planetary migration.

Despite these impressive successes, the observational picture of the planet abundance and their mass function is far from complete. This is partly caused by biases introduced by the detection techniques. The currently dominant radial-velocity method, despite ever improving sensitivity and temporal baselines, still favors massive planets in close-in orbits around solar type stars within about $100 \mathrm{pc}$ from the Sun. The transit method also favors the detection of close-in giant planets.

The microlensing technique, initially proposed by Mao \& Paczynski (1991) and whose prospects have been quantified first in more detail by Gould \& Loeb (1992), has now led to four reported detections (Bond et al. 2004; Udalski et al. 2005; Beaulieu et al. 2006; Gould et al. 2006). It has proved its capability to provide access to a new window for exoplanets, with masses down to an Earth-mass for ground-based searches and orbits between 1-10 AU mainly around host stars less massive than the Sun at several kpc distance. This is of special interest since most of stars in our Galaxy have masses less than $1 M_{\odot}$, so the planets of such stars, if common, may constitute the majority of Galactic exoplanetary systems. While the hunt for planets around low-mass stars still lags a bit behind the search for planetary companions to solar-type stars, the most recent microlensing discoveries of sub-Neptune mass (or super-Earth) planets (Beaulieu et al. 2006; Gould et al. 2006) indicate that such planets indeed are common, probably more common than any other class of exoplanets yet discovered. The rarity of cool Jovian companions to sub-solar mass stars (from 1-10 AU) seen in the microlensing data (Albrow et al. 2001; Gaudi et al. 2002; Tsapras et al. 2003; Snodgrass et al. 2004) on the other hand was recently complemented by radial velocity searches, deriving an upper limit of $<1 \%$ for the fraction of close-in (within $1 \mathrm{AU}$ ) Jovian M-dwarf planets (Endl et al. 2006).

These new observational constraints seem to be in line with predictions of planet formation theories. For example simulations done by Ida \& Lin (2005) and Laughlin et al. (2004) suggest that the formation of Jovian gas giants around M-dwarfs is inhibited, while planets less massive than Neptune can easily form. This is supported by recent microlensing results as one of the gas-giant planets discovered by microlensing was recently found to orbit a K-dwarf (Bennett et al. 2006), while the least massive planets discovered so far (OGLE 2005-BLG-390Lb, Gliese 476 b, Gliese $581 \mathrm{c}$ and d) are all orbiting M-dwarf stars. Disc instability formation models as advocated by Boss (2006) also are capable of explaining the preference of forming subNeptune mass planets rather than giant planets around low-mass stars.
In the study presented here we examine what constraints on a hypothetical Jovian planetary companion to the sub-Neptune mass planet detected in the microlensing event OGLE 2005BLG-390 (Beaulieu et al. 2006) can be derived from the photometric light curve data. This system contains a 3-10 $M_{\oplus}$ planet in orbit with semi major axis $a=2-5$ AU around an M-dwarf. We also estimate the probability of having detected an OGLE 2005-BLG-390Lb like planet in an idealized microlensing experiment and discuss in more details the claims made in Beaulieu et al. (2006) and Gould et al. (2006) that sub-Neptunes are common companions to low-mass stars.

\section{Basics of microlensing}

During a microlensing event, the light arising from a background star (the source star) is bent due to the gravitational field of an intervening planetary system (the lens) passing close to the observer line-of-sight. This results in a characteristic transient magnification of the source leading to a variation in the received flux, which constitutes the microlensing event light curve.

With $M$ denoting the total lens mass and $D_{\mathrm{S}}, D_{\mathrm{L}}$ the distances of source and lens from the observer respectively, the angular Einstein radius (Einstein 1936)

$\theta_{\mathrm{E}}=\sqrt{\frac{4 G M}{c^{2}}\left(\frac{D_{\mathrm{S}}-D_{\mathrm{L}}}{D_{\mathrm{L}} D_{\mathrm{S}}}\right)}$

defines the natural scale of gravitational microlensing. It equals the angular radius of the ring-shaped image of the source star which would occur for a perfect alignment between the observer, lens and source. With sources typically located in the Galactic Bulge and a lens lying at several kpc from us in the direction of the source, its linear scale at the lens distance $R_{\mathrm{E}}=D_{\mathrm{L}} \theta_{\mathrm{E}}$ corresponds to $\sim 1-10 \mathrm{AU}$, a range well suited for extrasolar planet hunting.

The transient brightening of the source (called magnification) due to a single lens can be fairly easily modeled and is described by an impact parameter $u_{0}$, with $u_{0} \theta_{\mathrm{E}}$ being the minimal angular distance between lens and source at time $t_{0}$, as well as a time-scale $t_{\mathrm{E}}$ which is the duration for the source to move by $\theta_{\mathrm{E}}$ on the sky. The total observed flux $F(t)$ is then expressed as the sum of the magnified source flux $A(t) \times F_{\mathrm{S}}$, with $A(t)$ being the magnification factor, plus the so-called blend flux $F_{\mathrm{B}}$ stemming from unresolved sources within the aperture, so that $F(t)=A(t) F_{\mathrm{S}}+F_{\mathrm{B}}$. Furthermore, one must take into account a pair of flux parameters $\left(F_{\mathrm{S}}, F_{\mathrm{B}}\right)$ per observing telescope.

A lens with a planet is usually modeled by a binary lens with an extreme mass ratio $\left(q<10^{-2}\right)$. The most striking characteristic of binary lenses when compared to single lenses is the occurrence of extended caustics, closed lines defined in the source plane where the magnification becomes infinite for point sources. However in practice the magnification remains finite since real sources have a finite extent. Nevertheless caustics mark regions of large magnification gradients. In the case of planetary lensing the area enclosed by these caustics (defining a kind of cross section) compared to the area covered by the Einstein ring disc is only a few percent at most, but if the source trajectory passes sufficiently close to or even traverses a caustic it can imprint a high signal-to-noise light curve signature, revealing the presence of the planet. Unfortunately this signal is only short-lived (ranging from hours to days for Earths to Jupiters) with respect to the complete event time-scales of weeks to months. This is the big observational challenge for teams like 

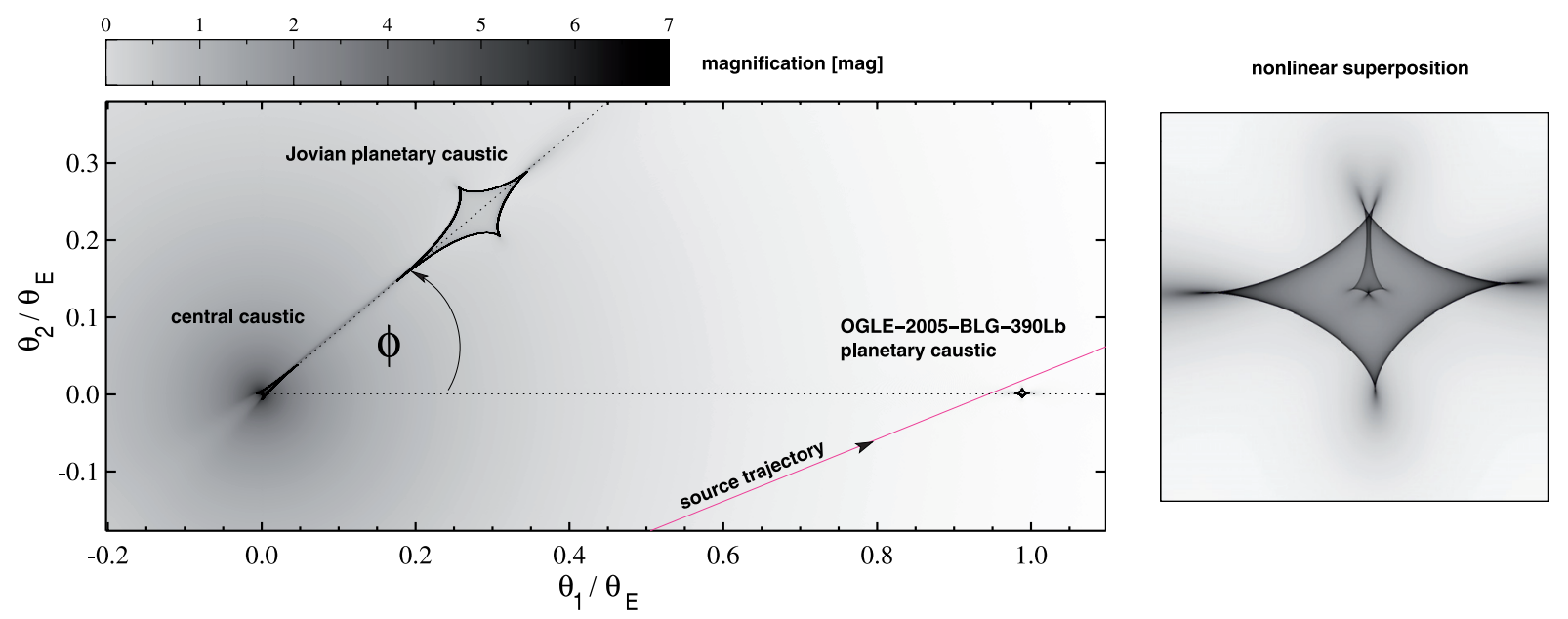

Fig. 1. The left panel of the figure displays a magnification map of a triple lens system configuration, consisting of a host star at the origin and two planets. The magnification is shown as function of the position angle $\left(\theta_{1}, \theta_{2}\right)$ on the sky. The darker the region the higher the magnification. The caustic contours have been over-plotted (black solid lines) for clarity. The small planetary caustic induced by OGLE 2005-BLG-390Lb is shown together with the best-fitting source track (Beaulieu et al. 2006, pink solid line). The larger planetary caustic on the upper left stems from a hypothetical additional Jovian companion, in this particular case with $q=2.0 \times 10^{-3}$ and $d=1.2$. Note that the actual positions of the planets do not coincide with the position of their planetary caustics. They lie on the dotted axes but outside the shown field of view, which scale was chosen to maximize the visibility of the caustics. Finally the right panel shows one of the rare configurations where the caustics of the two planets are close to the same position merging in a non-linear way, showing the limit of the linear superposition approximation.

PLANET/RoboNet ${ }^{2}, \mathrm{OGLE}^{3}, \mathrm{MOA}^{4}$ and MicroFUN ${ }^{5}$ to monitor microlensing events with a high sampling rate and not to miss these planetary "anomalies" in light curves which for most of the time are indistinguishable from a single-lens event.

The challenge on the modeling side is that the magnification of a binary lens cannot be expressed in a closed analytical form and its high-dimensional parameter space has an intricate $\chi^{2}$-surface on which the problem of parameter optimization is far from trivial. In addition to the single-lens model parameters the following ones are required: the planet-to-star mass ratio $q$, the lens separation $d$, with $d \times \theta_{\mathrm{E}}$ being the instantaneous angular distance of the planet from its host star, the impact angle $\alpha$ between the source trajectory and the binary lens axis. If the source is resolved by the lens, the angular source size $\theta_{*}$ and parameters describing the source surface profile also need to be considered.

\section{Detection efficiency of additional planets around OGLE 2005-BLG-390L}

Gaudi \& Sackett (2000) have presented an algorithm well-suited to compute the "efficiency" with which a binary companion to the lens can be revealed in an observed microlensing event. This algorithm applies for microlensing events where no clear deviation from a single point-mass lens can be seen in the light curve, i.e. events for which there is no evidence for a companion to the lens. Efficiency calculations as done in Albrow et al. (2000) or Gaudi et al. (2002) indeed were aimed to give limits on

\footnotetext{
2 http://planet.iap.fr

http://www.astro.livjm.ac.uk/RoboNet/

3 http://www. astrouw. edu.pl/ ogle

${ }^{4}$ http://www.phys.canterbury.ac.nz/moa

5 http://www-astronomy. mps.ohio-state. edu/ microfun
}

companions to lenses where no planets were found. Here we extend this method to compute the detection efficiency of multiple planetary systems, more precisely to put confidence limits on the presence of an hypothetical second planet in a system where one planet has already been detected. For similar reasons to the original method, the algorithm holds as long as there is no further clear signal in the light curve other than the already detected planet. We first outline the method before applying it specifically to OGLE 2005-BLG-390L.

As a natural extension of the method proposed by Gaudi \& Sackett (2000), we use a triple lens configuration to describe the discovered planet plus and additional companion, for which we want to compute detection efficiencies (i.e. calculating confidence limits on the latter's existence). Such a geometrical configuration is shown in the left panel of Fig. 1, where the parent star of the system is located at the origin and known OGLE 2005BLG-390Lb is situated on the horizontal axis on the right. A hypothetical additional planet with mass ratio $q$ is located at a distance $d$ from its parent star $\left(d=1.2, q=2 \times 10^{-3}\right.$ and $\phi=60^{\circ}$ in figure) with its planet-star axis subtending an angle $\phi$ with the $x$-axis. Note that with a planetary signal already detected, the trajectory of the source is known and fixed. We then compute detection efficiency on the second planet using the algorithm described below:

1. Compute the $\chi_{\text {ref }}^{2}$ of the model that best fits the already discovered planet,

2. Choose a given triple lens configuration with parameters $(d, q, \phi)$, where $\phi \in[0,2 \pi]$, and fit for the remaining parameters to compute the $\chi^{2}$ difference $\Delta \chi^{2}(d, q, \phi)=\chi^{2}(d, q, \phi)-$ $\chi_{\text {ref }}^{2}$,

3. Repeat step (2) until a dense sampling of $\phi$ is obtained for the probed lens configurations,

4. Repeat from step (3) until all the chosen lens configurations are probed.

The detection efficiency of an additional planet in the system is then given by the fraction of angles $\phi$ that produce a significant 
deviation in the light curve. By significant deviation, we mean an excess of $\chi^{2}$ of the probed lens model by an amount of $\chi_{\text {thresh }}^{2}$ relative to the single planetary model. More formally the detection efficiency is given by the formula:

$\varepsilon(d, q)=\int_{0}^{2 \pi} H\left(\Delta \chi^{2}(d, q, \phi)-\chi_{\text {thresh }}^{2}\right) \mathrm{d} \phi$,

$H$ being the Heaviside step function.

Apart from the computationally challenging sheer size of the parameter space of multiple lens systems another notable difficulty arises when the spatial extent of the source star has to be considered. Rather than integrating over the source surface, we make use of magnification maps that are much better designed for our purpose. A magnification map is obtained by shooting light rays from the observer through the lens and towards the source (Wambsganss 1997); assuming a constant spatial ray density in the plane containing the lens, its density in the plane where resides the source then maps the magnification as a function of its position. Such maps can easily include the extended source effects: The ray-shot maps just need to be convolved using the right size and brightness profile of the chosen source star (e.g. Kubas et al. 2005). A speed optimized version of the original ray-shooting algorithm is presented in Rattenbury et al. (2002). Figure 1 is an example of such a triple lens magnification map.

In general the source size in physical or Einstein units is not known and estimates rely on statistical Galactic models. The uncertainty of those parameters directly affects the uncertainties of the computed detection efficiencies (cf. Gaudi et al. 2002). The situation is much more favorable when, as in the case of OGLE 2005-BLG-390Lb, extended source effects are detected and strong constraints can be put on the source properties. Then it is straightforward to convolve a library of maps with the appropiate size and brightness profile of the source which as a welcomed byproduct reduces the to be explored parameter space.

Nevertheless this approach is still resource intensive since it requires computing a large number of magnification maps for the different probed characteristics $(d, q, \phi)$ of the second planet. However, in the case of OGLE 2005-BLG-390 we can simplify the problem with a fair approximation as follows. In fact, it has been shown that the planetary caustics in a multiple planetary system are independent to first order, as long as the projected planet positions do not overlap (Bozza 1999). However when the planetary caustics of the two planets are too close, non-linearities appear and the superposition principle is not valid anymore. This effect can be seen in the right panel of Fig. 1, where the two caustics are superposed in a non-linear way. Here the more massive hypothetical planet has been located only 0.05 fractions of $R_{\mathrm{E}}$ above OGLE 2005-BLG-390Lb causing the small caustic to flip its orientation by about 90 degrees and splitting two of its cusps. Hence, the superposition principle of the caustics is valid for most values of the angle $\phi$ and position $d$ of the additional planet, except when the caustics coincide. We make the choice here to compute only binary lens maps and use the superposition principle. By doing so, we save around two orders of magnitude of computational time to create the map library (each magnification map requires few hours of computation with a usual CPU).

To deal with the rare configurations when the non-linearities appear, one choice would be to subtract the best-fit binary-lens model from the original OGLE 2005-BLG-390Lb light curve and then add the residuals to form a new light curve based on the underlying single-lens model. This new "single-lens" light curve then serves as a reference to which a series of binary-lens models are fitted according to Gaudi \& Sackett (2000). However we prefer here to adopt a more conservative choice, by cutting out the data from the region where the signal of OGLE 2005BLG-390Lb resides (i.e. MHJD between 3592.50 and 3593.11) prior to the computations. While with both options one cannot remove the intrinsic non-linearity at the common position of the caustics, the uncertainties they introduce are small and in any case of similar order of magnitude.

We have computed a $(d, q)$ magnification map library of lens configurations spanning nine different mass ratios $(q=$ $\left.5.6 \times 10^{-4}-10^{-2}\right)$ and covering sixteen lens separations $(d=$ $0.1-3.3$ ), convolved with the source brightness profile adopted by Beaulieu et al. (2006). The choice of the grid is motivated by the goal to cover the theoretical range of Jovian type planets probed in the simulations of Ida \& Lin (2005), and also to ensure a good coverage of the so-called lensing zone, the range of lens separations, where microlensing is most sensitive to planets. For each $(d, q)$ configuration we have computed 200 models with $\phi_{k}=2 k \pi / 200$, i.e. in total $200 \times 16 \times 9=28800$ models.

At each $(d, q)$-grid point and for each angle $\phi \in[0,2 \pi]$ we then compute the least-square measure $\chi^{2}(d, q, \phi)$ optimized over the remaining free parameters: minimum impact parameter $u_{0}$, time of maximum magnification $t_{0}$, Einstein radius crossing time $t_{\mathrm{E}}$, as well as the source fluxes $F_{\mathrm{S}}{ }^{i}$ and blending fluxes $F_{\mathrm{B}}{ }^{i}$ for each of the 6 different telescope data sets used. These optimized parameters are "free" in the sense that we allow them to vary within the error bars of the best-fitting values derived in Beaulieu et al. (2006). This flexibility minimizes numerical noise in the calculations without violating the constraints given by the best model. We checked that the results are consistent with fixing the parameters to their best-fitting values and that using non-bounded parameters yields unphysical results. To carry out the optimization we use a genetics algorithm (Charbonneau 1995), which naturally provides the capability to bound parameters and has been shown to explore the intricate parameter space of binary lenses more efficiently than classical gradient optimization techniques (Kubas 2005; Cassan 2005).

A given choice of parameters $(d, q, \phi)$ is considered to produce a significant deviation if $\Delta \chi^{2}=\chi^{2}(d, q, \phi)-\chi_{\text {ref }}^{2}$ exceeds a threshold value $\chi_{\text {thresh }}^{2}$. We set $\chi_{\text {thresh }}^{2}=60$, which we find robust enough to avoid false detections arising from statistical fluctuations or unrecognized low-level systematics and to be consistent with earlier detection efficiency studies (Gaudi et al. 2002; Yoo et al. 2004).

Figure 2 shows contours of the detection efficiency $\varepsilon(d, q)$ as a function of the dimensionless model parameters $d$ and $q$, where $d \times \theta_{\mathrm{E}}$ is the angular separation of the planet from its host star while $q$ is the planet-to-star mass ratio. Calculations using an algorithm based on a method described in Rhie et al. (2000) were also used to compute detection efficiencies, with comparable results.

The obtained detection efficiency constraints are admittedly rather weak. However this is not a flaw of the data or analysis but mainly due to the intrinsic nature of a low magnification event. In fact, Gaudi \& Sackett (2000) have shown that the detection efficiency of a microlensing event is strongly dependent on the minimum parameter $u_{0}$ of the underlying single lens model: the smaller $u_{0}$, the higher the peak magnification and the higher the detection efficiency. This can be understood as follows.

As depicted in Fig. 1, in a planetary binary lens scenario one has to differentiate between the central caustic, always located close to the primary lens (star), and the planetary caustics, the location of which strongly depends on the projected star-planet 


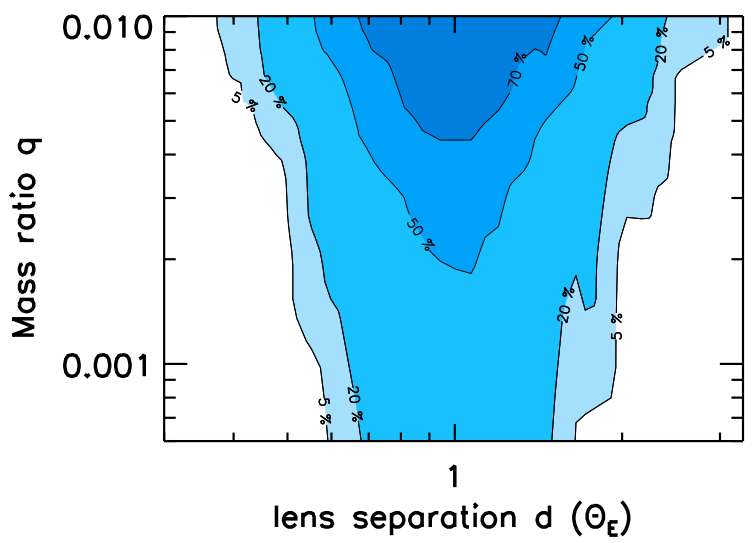

Fig. 2. Detection efficiency $\varepsilon(d, q)$ as a function of angular planet-to-star separation $d \times \theta_{\mathrm{E}}$ and planet-to-star mass ratio $q$. These dimensionless efficiencies present the raw outcome of our computation prior to the convolution given in Appendix A where we derive the physical detection efficiency values as functions of planet mass and orbital radius.

separation $d$. While as stated earlier the planetary caustics are basically mutually independent from each other, the central caustic is affected by any companion to the primary lens and thus also especially sensitive to multiple planet systems. To probe the central caustic sufficiently small impact parameters $u_{0}$, respectively high magnifications, are required. Griest \& Safizadeh (1998) showed that for $u_{0} \rightarrow 0$ the planet detection efficiency goes to one, unless finite-source effects prevent the detection of the least massive planets around the highest peaks for larger sources. The drawback of high magnification central caustic events however is that they are much harder to model and sometimes plagued by a close-wide binary ambiguity (Dominik 1999; Kubas et al. 2005). In the OGLE 2005-BLG-390 event the impact parameter is too large to explore the central caustic, which explains the calculated restricted sensitivity to additional planets.

\section{Limits on Jovian companions to OGLE 2005-BLG-390L}

While $\varepsilon_{\mathrm{m}}(d, q)$ is the most straightforward constraint we can get from modeling, one would like to infer statements on the underlying physical parameters, the planet's orbital axis $a$ and its mass $m$. The planet mass and the mass ratio are linked by $m=q \times M$, with $M$ being the host star mass. The physical (instantaneous) lens separation projection $r$ is given by $r=d \times D_{\mathrm{L}} \times \theta_{\mathrm{E}}$. Adopting the median values determined by Beaulieu et al. (2006), namely $D_{\mathrm{S}} \simeq 8.0 \mathrm{kpc}, D_{\mathrm{L}} \simeq 6.6 \mathrm{kpc}$ and $M \simeq 0.22 M_{\odot}$, we have:

$$
\begin{aligned}
& m=q \times M \simeq q \times 0.22 M_{\odot} \\
& r=d \times D_{\mathrm{L}} \theta_{\mathrm{E}} \simeq d \times 1.35 \mathrm{AU} .
\end{aligned}
$$

However, without a proper measurement of both the source size and the parallax in Einstein radii (e.g. Ghosh et al. 2004; Jiang et al. 2004), the mass $M$ of the host star OGLE 2005BLG-390L and the Einstein radius are distributed in probability over a finite range, where both distributions are correlated, and moreover, the orbital axis follows from $r$ by a stochastic orbital de-projection. Therefore, one needs to take these distributions into account for expressing the detection efficiency as function of $(a, m)$. Assuming circular orbits and the stellar mass function from Chabrier (2003), we use the implementation of a

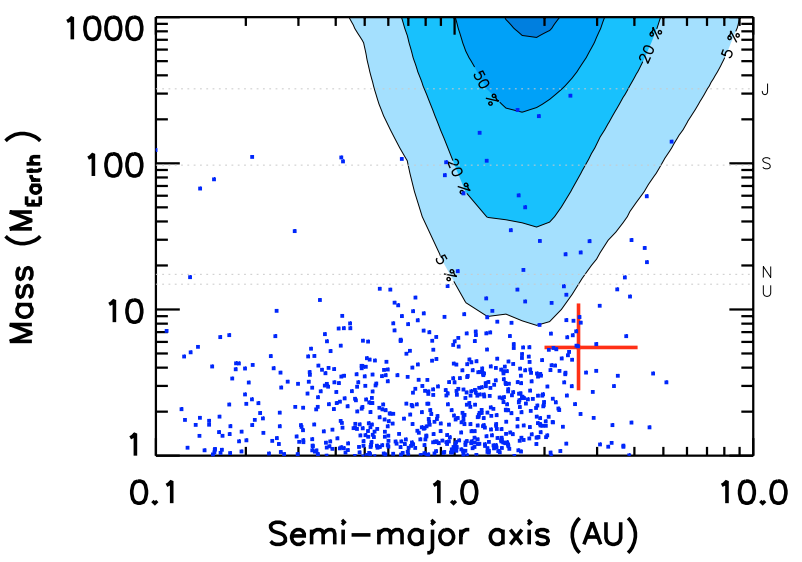

Fig. 3. Detection efficiency $\tilde{\varepsilon}_{\mathrm{p}}(a, m)$ for additional planets orbiting OGLE 2005-BLG-390L as function of orbital separation $a$ and planet mass $m$, where contours at 5,20,50 and 70\% are shown. The cross marks the median values for the properties of OGLE 2005-BLG-390Lb along with $68.3 \%$ confidence intervals and the dashed horizontal lines mark the masses of Jupiter (J), Saturn (S), Neptune (N) and Uranus (U) for comparison. The blue dots represent the predicted final distribution of a seed of 20000 planetary cores around an M-dwarf of $0.2 M_{\odot}$ resulting from a core-accretion model assuming inefficient migration (taken from Fig. 9b of Ida \& Lin 2005).

Galactic model by Dominik (2006) in order to derive detection efficiency values $\varepsilon(a, m)$. The details of this procedure are given in Appendix A.

The resulting detection efficiency diagram is presented in Fig. 3, which shows a contour plot of $\varepsilon(d, q)$ together with OGLE 2005-BLG-390Lb (red error bar cross). It basically tells us about confidence limits on additional companions to OGLE 2005-BLG-390Lb: values of $\varepsilon(d, q)$ close to unity rule out the possibility of such an additional planet at $(a, m)$, while values close to zero mean that no conclusion can be drawn about the multiplicity of OGLE 2005-BLG-390L.

The strongest constraints from the diagram are placed in the Jovian-mass regime, where $\varepsilon(a, m)>50 \%$. We find that planets more massive than $1 M_{\mathrm{J}}$ in the orbital range 1.1-2.3 AU have a detection efficiency in excess of $50 \%$, while planets above $3 M_{\mathrm{J}}$ in orbits between 1.5 and 2.2 AU would have revealed their existence with a probability of more than $70 \%$. Unfortunately, our data cannot tell us about possible Pegasid planets (giant planets orbiting at fractions of $1 \mathrm{AU}$ ), which could exist in OGLE 2005BLG-390L.

On the same Fig. 3, we have plotted the results of MonteCarlo simulations by Ida \& Lin (2005), producing the final evolution stage of a seed of 20000 planetary embryos, uniformly distributed in $\log a$ (from 0.1 to $100 \mathrm{AU}$ ), around a host star with $M=0.2 M_{\odot}$, similar to OGLE 2005-BLG-390L. In principle, such simulations are valid when considering a single planet, whereas we consider the case of two planets. We therefore choose among the available models of Ida \& Lin (2005) one for which the planet migration process is very inefficient and so practically assume that the planets were formed quasi-in situ. This allows us to use the efficiency diagram to compare our observation to this planet formation model. In the chosen planet formation scenario - model from Fig. 9b of Ida \& Lin (2005) migration is strongly suppressed, the cores have more time for accretion, more gas giants can form and planets (blue points on Fig. 3) stay close to their orbital birth places. Two main predictions can be read out from their simulation. Firstly, sub-NeptuneM-dwarf planets should vastly outnumber gas-giant-M-dwarf 
ones. In the case of an $0.2 M_{\odot} \mathrm{M}$-dwarf the formation of a subNeptune planet between 1 and $14 M_{\oplus}$ in the orbital range of 1-10 AU is about 1200 times more likely than forming Jovian planets with $0.5-1 M_{\mathrm{J}}$ in the same orbital range. Secondly if giant planets form they are unlikely to become more massive than $\sim 1 M_{\mathrm{J}}$.

In the framework of disc instability planet formation theory, the preference for sub-Neptune planets can also be explained by photo evaporation of the gas envelopes of giant protoplanets, as recently pointed out by Boss (2006). However in this case there are no obvious reasons for postulating a gap in the planetary mass function between Jupiter and Neptune masses.

We note that also around the other known low-mass planet found by microlensing OGLE 2005-BLG-169Lb, which hoststar is most likely a K-dwarf, there were no traces found in the data of additional Jovian companions in the orbital range between about 0.5 and $14 \mathrm{AU}$ (Gould et al. 2006).

The current sample of known planets around M- and $\mathrm{K}$-dwarfs is still too small to distinguish between the alternative scenarios of planet formation and migration. However, just a few more detections are likely to add valuable further information.

\section{On the detection of OGLE 2005-BLG-390Lb-like planets}

Beaulieu et al. (2006) and Gould et al. (2006) have stated that their respective detections of the low-mass planets OGLE 2005BLG-390Lb and OGLE 2005-BLG-169Lb imply a large abundance of their siblings. The sensitivity of a microlensing light curve to the presence of planets depends strongly on, besides data sampling and quality, the event magnification and to some extent on the event time-scale and source size as well. Increasing the angular size of the source has, for instance, the dual effect of increasing the duration of the planetary signal while, at the same time, decreasing its amplitude. The latter effect ultimately restricts the capabilities for detection of planets to $\sim 1 M_{\oplus}$ for giant sources and to $\sim 0.1 M_{\oplus}$ for turnoff stars in the Galactic bulge (Bennett \& Rhie 1996).

An accurate determination of planet abundances therefore requires an analysis of the detection efficiency of a representative sample of the whole experimental data set and a comparison with the actual detections. For the PLANET campaign, this will be worked out in an upcoming study (Cassan et al. 2008). Here, we run simulations to derive estimates of the probability for detection of sub-Neptune or Jovian planets based on simplified assumptions.

We choose to pick impact parameters $u_{0}$ from a uniform distribution, which is close to what is actually realized in nature; however, only those events with a magnification above a characteristic threshold will be observable. Neglecting blending, we sample $u_{0} \in[0,1]$ in 100 equally spaced steps, corresponding to magnifications $A>1.34$. While this means that we practically cover the whole range of magnifications in the alerted and followed-up microlensing events, we neglect the preference for higher magnifications in the follow-up campaigns.

In Sect. 3 we made use of a $\Delta \chi^{2}$-based criterion for ruling out additional planets in the OGLE 2005-BLG-390L system. While in principle such a criterion works for detections as well we note that in practice one often finds that even if this criterion is fulfilled, a lack in coverage and/or precision makes it impossible to characterize the planetary model unambiguously. Unlike a rejection a convincing detection has to meet stronger criteria (as, for instance, not showing systematic trends and having a sufficient number of data points Vermaak 2000), which are not reflected in a criterion solely based on $\chi^{2}$. In other words we want to avoid cases in which planets are detectable but not characterizable, respectively cannot be distinguished from non-planetary solutions.

To minimize the effect of this caveat on our simulation we therefore adopt a more demanding criterion to calculate the efficiency to discover OGLE 2005-BLG-390Lb-like planets. For a "discovery", we demand that the planetary signal amplitude exceeds $2 \%$ for at least 15 measurements, assuming an hourly sampling and a photometric accuracy of $1 \%$. Neglecting nonwhite noise this criterion then translates into $\Delta \chi^{2}=60$, i.e. is consistent with the detection threshold chosen in Sect. 3. This criterion ensures that the planetary light curve signature is also well sampled, which is essential for a proper characterization of the lens system. For comparison we also compute the efficiencies for the following set of criteria: the signal amplitude should exceed $2 \%$ for at least 10 and 25 measurements.

Since the detection efficiency reaches a maximum in the socalled "lensing zone", corresponding to a star-planet separation range of $0.62 \leq d \leq 1.62$, we can restrict our sampling to the time range $\left[t_{0}-t_{\mathrm{E}}, t_{0}+t_{\mathrm{E}}\right]$, as most microlensing campaigns do, which ensures being sensitive to planets at these separations. To avoid border effects, we however draw the simulated light curves from magnification maps spanning $t_{0} \pm 1.2 t_{\mathrm{E}}$. For the source star, we adopt an angular size $\theta_{*}=9.6 \times 10^{-3} \theta_{\mathrm{E}}$ (which corresponds to $\theta_{*}=5.25 \mu$ as or a physical radius $R_{\star} \sim 10 R_{\odot}$ in the case of OGLE 2005-BLG-390Lb).

As in Sect. 3, we use the ray-shooting technique for calculating magnification maps. While we adopt fixed mass ratios of $q=7.6 \times 10^{-5}$, which is the value for OGLE 2005-BLG-390Lb, and $q=4.3 \times 10^{-3}$, which corresponds to $M=1 M_{\mathrm{J}}$ if the mass of OGLE 2005-BLG-390Lb is 5.5 $M_{\oplus}$, we use a grid for the lens separation $d=0.1-5.0$. Again as in Sect. 3, we compute the fraction of detections made over this grid assuming that every lens has such a planet by averaging over the impact angle and impact parameter.

In Fig. 4 the derived efficiencies are shown as a function of the projected separation $d$, for the selected mass ratios and time scale, $t_{\mathrm{E}}=11.0$ as measured for OGLE 2005-BLG-390. For criterion $C_{2}$ (more than 15 deviating points) at the separation parameter for OGLE 2005-BLG-390Lb, $d=1.61$, the detection efficiency for an OGLE 2005-BLG-390Lb-like mass ratio of $q=7.6 \times 10^{-5}$ is about $1 \%$, whereas it becomes about $50 \%$ for $q=4.3 \times 10^{-3}$, resembling a Jupiter-mass planet around the same host star, i.e. the sensitivity to Jupiters in this case is, as was reported by Beaulieu et al. (2006), about 50 times higher than it is to sub-Neptunes. Unsurprisingly the sensitivity to Jovians only shows a weak dependance on the applied detection criteria, since the majority of the associated anomalies are strong enough to be detected even by our most stringent detection demands. The sensitivity for OGLE 2005-BLG-390Lb-like mass ratio on the other hand is much more affected by the choice of the detection threshold and, when integrated over the lensing zone, ranges from $\sim 0.5-3 \%$ for criteria $C_{3}$ to $C_{1}$. For comparison we have also computed and plotted the detection efficiency using a pure $\Delta \chi^{2}$ threshold with $\Delta \chi^{2}=60,220,400$, assuming the same light curve sampling rate (hourly) and photometric accuracy $(1 \%)$. We note that whereas the efficiencies for Jovian planets again remain rather unaffected at levels of $\sim 50-60 \%$, the detection efficiencies drops to $2-5 \%$ for OGLE 2005-BLG390Lb-like companions. In fact, a criterion assuming $\Delta \chi^{2}=60$ is much less demanding that the $C_{2}$ criterion, since the latter rejects a lot of anomalies which are too short lived to pass the minimal sampling requirement of 15 points. 


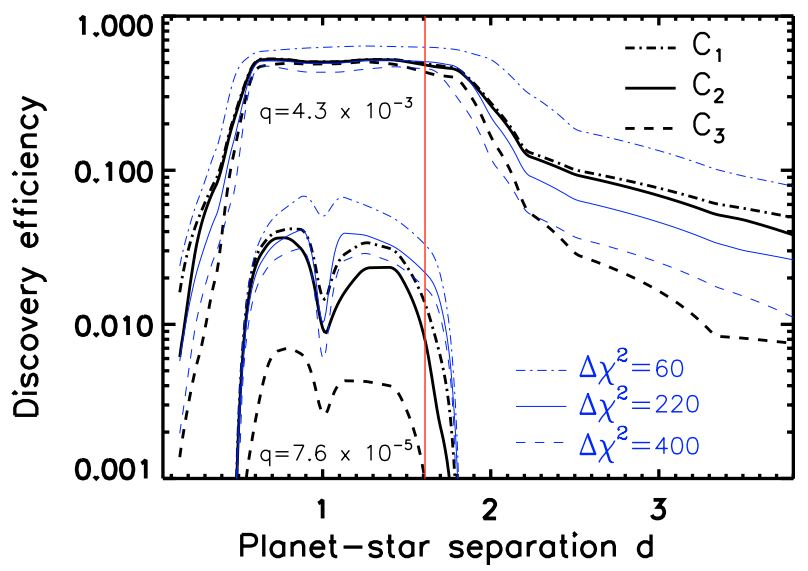

Fig. 4. Efficiency of detecting a super-Earth planet with the mass ratio of OGLE 2005-BLG-390Lb ( $q=7.6 \times 10^{-5}$, lower set of curves), and for a giant planet with a Jupiter/Sun mass ratio $\left(q=4.3 \times 10^{-3}\right.$, upper set of curves) as a function of the projected planet-star separation $d$. The bold black curves have been computed assuming an hourly sampled light curve with $1 \%$ photometric accuracy, and demanding that the planetary signature amplitude exceeds $2 \%$ for at least 10 measurements (dotted line, indexed as $C_{1}$ ), 15 and 25 measurements (solid line, $C_{2}$ and dashed line, $C_{3}$ ). For comparison efficiencies for the same sampling rate and accuracy but using a pure $\Delta \chi^{2}$ threshold are plotted as well, with $\Delta \chi^{2}=60,220,400$ (blue thin lines). The considered impact parameters are drawn uniformly from $u_{0} \in[0,1]$. ased on the parameters found for the lens scenario of OGLE 2005-BLG-390, a time scale of $t_{\mathrm{E}}=11.0$ days was adopted, as well as a source size of $\sim 10 R_{\odot}$. The vertical line marks the actual separation of OGLE 2005-BLG-390Lb.

Averaged over the lensing zone one finds sensitivity ratios between Jupiters and sub-Neptunes of up to 25 for OGLE 2005BLG-390-like time scales of $t_{\mathrm{E}}=11.0$ days. For time scales of around $t_{\mathrm{E}}=22.4$ days, which is the median time scale of events followed up by planet, the simulations yield that Jovians are about 10 times easier to detect that sub-Neptunes.

However, there are some notable differences between the assumptions for our simulation and the real campaigns. First, even in dense telescope networks, weather and technical losses are unavoidable and a continous data coverage can not always be achieved. Secondly the peak magnification of OGLE 2005BLG-390Lb ( $A_{\mathrm{o}} \sim 3$, corresponding to $\left.u_{0}=0.36\right)$ is one of the lowest among the events monitored by PLANET/RoboNet. Follow-up teams preferentially monitor events with larger magnifications, thereby preferring smaller $u_{0}$. As stated at the end of Sect. 3, the detection efficiency usually increases with the magnification and therefore the calculated efficiency for $u_{0}$ uniformly chosen in $[0,1]$ underestimates the chance to detect less-massive planets in high magnification events. Moreover, campaigns like PLANET/RoboNet intensify the sampling over the peak region, which significantly enhances the chance to detect and characterize super-Earths as compared to a standard sampling scheme. On the other hand, our simulations overestimate the chance to detect OGLE 2005-BLG-390Lb-like in low magnification events, due to the increased anomaly durations when a giant source sweeps over a planetary caustic, whereas a main sequence source star would on the contrary produce a signal with higher amplitude but shorter duration.

Despite its simplicity, our simulation however shows robustly that in any case OGLE 2005-BLG-390Lb-like planets have a modest but not negligible probability for discovery, while Jovian-type planets should be found much more easily. With OGLE 2005-BLG-390Lb already being the third reported detection of a planet by microlensing (Beaulieu et al. 2006) after two Jovian planets, we therefore have the first observational evidence for the suggestion that sub-Neptunes are common around M-dwarfs and much more frequent than Jovian companions. The detection of another super-Earth by Gould et al. (2006) gives further support to this finding.

\section{Summary and conclusions}

We have re-examined the photometric data on OGLE 2005BLG-390Lb to look for traces of additional Jovian companions, finding that additional planets more massive than $1 M_{\mathrm{J}}$ and in orbits of $\sim 1.1-2.3$ AU would have caused a detectable signal in more than $50 \%$ of the cases, which however was not observed. Planets with masses of $3 M_{\mathrm{J}}$ and above between $\sim 1.5-2.2 \mathrm{AU}$ would have revealed themselves in the data with a probability of $70 \%$.

Planet formation models based on sequential accretion processes by Ida \& Lin (2005) predict that the creation of gas giants is strongly suppressed around M-dwarfs for practically the whole range of their model parameters. In agreement with such theoretical predictions we do not find in our data any indication of an additional companion to OGLE 2005-BLG-390Lb in the Jovian mass regime, however we are not able to definitely exclude this possibility.

Assuming the natural unfiltered uniform distribution of lenssource impact parameters above an un-blended magnification threshold of $A=1.34$ and a simple sampling pattern, we find a detection probability for an OGLE 2005-BLG-390Lb-like planet in events involving giant source stars of $1-3 \%$ at its angular separation of 1.61 times the angular Einstein radius $\theta_{\mathrm{E}}$, whereas the average over the lensing zone, i.e. separations between 0.62 and $1.62 \theta_{\mathrm{E}}$, becomes $2-5 \%$. With detection probabilities of a few percent, our discovery of OGLE 2005-BLG-390Lb provides the first observational evidence in support of the prediction by current planet formation theories that sub-Neptune mass planets are common.

Acknowledgements. We are very thankful to S. Ida and D.N.C. Lin for providing their simulation results and permission to incorporate them in Fig. 3. We express our deepest gratitude to the OGLE and MOA collaborations for continuing to provide us with alerts on ongoing microlensing events from which we choose our targets. Part of the work was supported by an ESO DGDF grant for D.K. and a University of Tasmania IRIG grant for D.K. and A.C. We especially thank the University of Tasmania for granting us access to their TPAC supercomputer facilities where part of the calculations were carried out. This work was also supported by the French HOLMES ANR grant. KHC's work as performed under the auspices of the US DOE, LLNL in part under Contract W-7405-Eng-48 and in part under Contract DE-AC52-07NA27344. We also would like to thank the anonymous referee for his constructive comments. Furthermore D.K. and A.C. would like to thank C. Mordasini for inspiring discussions on planet formation theory.

\section{Appendix A: Conversion to physical lens parameters}

The parameters that can be extracted from the observed light curve are not sufficient for directly determining the properties of the planet and its host star, such as their masses, the orbital radius and period, as well as their distance. However, as discussed by Dominik (2006), one can derive probability densities by means of Bayes' theorem under the assumption of a mass spectrum for the lens stars, the lens and source distance, following the spatial mass density of stars in the Milky Way, and their velocity 
distribution. The planet itself is characterized by the planet-tostar mass ratio $q=m / M$ and the separation parameter $d$, where $d \theta_{\mathrm{E}}$ is the instantaneous angular separation between the planet and its host star. All further information relies on the time-scales $t_{\mathrm{E}}=\theta_{\mathrm{E}} / \mu$ and $t_{\star}=\theta_{\star} / \mu$, during which the source moves by the angular Einstein radius $\theta_{\mathrm{E}}$ or its own angular radius $\theta_{\star}$ relative to the lens on the sky, where $\mu$ denotes its relative proper motion. With $\theta_{\star}$ determined from the source magnitude and color, one obtains $\mu$ and $\theta_{\mathrm{E}}$.

With $D_{\mathrm{L}}$ and $D_{\mathrm{S}}$ denoting the lens or source distance, respectively, let us define fractional distances $x=D_{\mathrm{L}} / D_{\mathrm{S}}$ and $y=D_{\mathrm{S}} / R_{\mathrm{GC}}$, where $R_{\mathrm{GC}}=(7.62 \pm 0.32) \mathrm{kpc}$ is the distance to the Galactic center (Eisenhauer et al. 2005). If one ignores selection effects for the source stars related to their luminosity function as well as extinction, the probability of finding these between $D_{\mathrm{S}}$ and $D_{\mathrm{S}}+\mathrm{d} D_{\mathrm{S}}$ becomes proportional to $D_{\mathrm{S}}^{2} \rho_{\mathrm{S}}\left(D_{\mathrm{S}}\right)$, where $\rho_{\mathrm{S}}\left(D_{\mathrm{S}}\right)$ denotes the volume mass density of the source stars, while $\rho_{\mathrm{L}}\left(D_{\mathrm{L}}\right)$ denotes the volume mass density of the lenses.

We further assume that the lens star mass spectrum $\Phi_{M / M_{\odot}}\left(M / M_{\odot}\right)$ stretches from $M_{\min }$ and $M_{\max }$ and does not depend on the lens distances, while we consider the distribution of the effective lens velocity $v=D_{\mathrm{L}} \mu$ to depend on both the lens and source distance. With a characteristic velocity $v_{\mathrm{c}}$, the dimensionless velocity parameter $\zeta=v / v_{\mathrm{c}}$ is distributed as $\Phi_{\zeta}(\zeta, x, y)=v_{\mathrm{c}} \Phi_{v}\left(v_{\mathrm{c}} \zeta, x, y\right)$.

With

$$
\begin{aligned}
\theta_{\mathrm{E}, \odot} & =2 \sqrt{\frac{G M_{\odot}}{c^{2} R_{\mathrm{GC}}}}=1030\left(\frac{R_{\mathrm{GC}}}{7.62 \mathrm{kpc}}\right)^{-1 / 2} \mu \mathrm{as} \\
\eta_{\theta_{\mathrm{E}}} & =\frac{\theta_{\mathrm{E}}}{\theta_{\mathrm{E}, \odot}}=9.68 \times 10^{-4}\left(\frac{\theta_{\mathrm{E}}}{1 \mu \mathrm{as}}\right)\left(\frac{R_{\mathrm{GC}}}{7.62 \mathrm{kpc}}\right)^{1 / 2} \\
\eta_{\mu} & =\frac{\mu R_{\mathrm{GC}}}{2 v_{\mathrm{c}}}=0.0660\left(\frac{\mu}{1 \mu \mathrm{as} \mathrm{d}^{-1}}\right)\left(\frac{R_{\mathrm{GC}}}{7.62 \mathrm{kpc}}\right),
\end{aligned}
$$

the measured values of $\theta_{\mathrm{E}}$ and $\mu$ determine the detection efficiency as function of the projected separation $r=d D_{\mathrm{L}} \theta_{\mathrm{E}}$ and the planet mass $m$ as

$$
\begin{aligned}
& \varepsilon_{r, m}(r, m)=\int_{\min \left(m / M_{\odot}, M_{\min } / M_{\odot}\right)}^{M_{\max } / M_{\odot}} \int_{0}^{\infty} \\
& \times \varepsilon_{d, q}\left[\frac{r}{y R_{\mathrm{GC}} \eta_{\theta_{\mathrm{E}}} \theta_{\mathrm{E}, \odot}}\left(\frac{y \eta_{\theta_{\mathrm{E}}}^{2}}{M / M_{\odot}}+1\right), \frac{m / M_{\odot}}{M / M_{\odot}}\right] \\
& \times p_{M / M_{\odot}}\left(M / M_{\odot}, y ; \eta_{\theta_{\mathrm{E}}}, \eta_{\mu}\right) \mathrm{d} y \mathrm{~d}\left(M / M_{\odot}\right),
\end{aligned}
$$

where $p_{M / M_{\odot}}$ denotes the probability density of the mass of the primary, which reads

$$
\begin{aligned}
& p_{M / M_{\odot}}\left(M / M_{\odot}, y ; \eta_{\theta_{\mathrm{E}}}, \eta_{\mu}\right)=\frac{R_{\mathrm{GC}}^{2}}{N_{0} M_{\odot}^{2}} f_{y}(y) y^{9 / 2} \eta_{\mu} \eta_{\theta_{\mathrm{E}}}^{2} \\
& \times \frac{\left(M / M_{\odot}\right)^{3}}{\left(y \eta_{\theta_{\mathrm{E}}}^{2}+M / M_{\odot}\right)^{5}} \Phi_{M / M_{\odot}}\left(M / M_{\odot}\right) \\
& \times \Phi_{\zeta}\left(\frac{2 y \eta_{\mu}\left(M / M_{\odot}\right)}{y \eta_{\theta_{\mathrm{E}}}^{2}+M / M_{\odot}}, \frac{M / M_{\odot}}{y \eta_{\theta_{\mathrm{E}}}^{2}+M / M_{\odot}}, y\right) \\
& \times \rho_{\mathrm{L}}\left(\frac{M / M_{\odot}}{y \eta_{\theta_{\mathrm{E}}}^{2}+M / M_{\odot}}\right) \rho_{\mathrm{S}}\left(y R_{\mathrm{GC}}\right),
\end{aligned}
$$

where $N_{0}=\iint p_{M / M_{\odot}}\left(M / M_{\odot}, y ; \eta_{\theta_{\mathrm{E}}}, \eta_{\mu}\right) \mathrm{d} y \mathrm{~d}\left(M / M_{\odot}\right)$.
The function $f_{y}(y)$ denotes a prior for the source distance, for which we adopted

$f_{y}(y)=\frac{1}{\sqrt{2 \pi} \sigma_{y}} \exp \left\{-y^{2} \frac{\left[\ln y-\ln y^{(0)}\right]^{2}}{2 \sigma_{y}^{2}}\right\}$

so that $\ln y$ follows a normal distribution around $\ln y^{(0)}$ with standard deviation $\sigma_{\ln y}=\sigma_{y} / y$.

With the assumption of circular orbits, de-projection assuming random orientation and phase, finally yields the detection efficiency as function of the orbital radius $a$ and the planet mass $m$ as

$\varepsilon_{a, m}(a, m)=\int_{0}^{1} \varepsilon_{\hat{r}, m}\left(\sqrt{1-\hat{w}^{2}} a, m\right) \mathrm{d} \hat{w}$.

For the mass and velocity distribution of the Milky Way, we follow Dominik (2006) in adopting a double-exponential disk and a barred bulge as well as mass spectra for disk and bulge lenses discussed by Chabrier (2003).

For OGLE-2005-BLG-390, we inferred $t_{\mathrm{E}}=11.0 \mathrm{~d}$ and $t_{\star}=$ $0.28 \mathrm{~d}$, so that with $\theta_{\star}=5.25 \mu \mathrm{as}$, one finds $\mu=18.6 \mu \mathrm{as} \mathrm{d}^{-1}$ and $\theta_{\mathrm{E}}=205 \mu \mathrm{as}$. Typing of the source by Beaulieu et al. (2006), yielded $D_{\mathrm{S}}^{(0)}=8.0 \mathrm{kpc}$ and $\sigma_{D_{\mathrm{S}}}=2.0 \mathrm{kpc}$.

\section{References}

Albrow, M. D., Beaulieu, J.-P., Caldwell, J. A. R., et al. 2000, ApJ, 535, 176 Albrow, M. D., An, J., Beaulieu, J.-P., et al. 2001, ApJ, 556, L113 Alibert, Y., Baraffe, I., Benz, W., et al. 2006, A\&A, 455, L25 Beaulieu, J.-P., Bennett, D. P., Fouque, P., et al. 2006, Nature, 439, 437 Bennett, D. P., \& Rhie, S. H. 1996, ApJ, 472, 660

Bennett, D. P., Anderson, J., Bond, I. A., Udalski, A., \& Gould, A. 2006, ApJ, 647, L171

Bond, I. A., Udalski, A., Jaroszyński, M., et al. 2004, ApJ, 606, L155

Boss, A. P. 2006, ApJ, 644, L79

Bozza, V. 1999, A\&A, 348, 311

Cassan, A. 2005, Ph.D. Thesis, Université Pierre et Marie Curie - Paris 6 Cassan, A., Kubas, D., Dominik, M., et al. 2008, in preparation

Chabrier, G. 2003, PASP, 115, 763

Charbonneau, P. 1995, ApJS, 101, 309

Dominik, M. 1999, A\&A, 349, 108

Dominik, M. 2006, MNRAS, 367, 669

Einstein, A. 1936, Science, 84, 506

Eisenhauer, F., Genzel, R., Alexander, T., et al. 2005, ApJ, 628, 246

Endl, M., Cochran, W. D., Kürster, M., et al. 2006, ApJ, 649, 436

Gaudi, B. S., \& Sackett, P. D. 2000, ApJ, 528, 56

Gaudi, B. S., Albrow, M. D., An, J., et al. 2002, ApJ, 566, 463

Ghosh, H., DePoy, D. L., Gal-Yam, A., et al. 2004, ApJ, 615, 450

Gould, A., \& Loeb, A. 1992, ApJ, 396, 104

Gould, A., Udalski, A., An, D., et al. 2006, ApJ, 644, L37

Griest, K., \& Safizadeh, N. 1998, ApJ, 500, 37

Ida, S., \& Lin, D. N. C. 2004, ApJ, 616, 567

Ida, S., \& Lin, D. N. C. 2005, ApJ, 626, 1045

Jiang, G., DePoy, D. L., Gal-Yam, A., et al. 2004, ApJ, 617, 1307

Kennedy, G. M., Kenyon, S. J., \& Bromley, B. C. 2006, ApJ, 650, L139

Kubas, D. 2005, Ph.D. Thesis, University of Potsdam

Kubas, D., Cassan, A., Beaulieu, J. P., et al. 2005, A\&A, 435, 941

Laughlin, G., Bodenheimer, P., \& Adams, F. C. 2004, ApJ, 612, L73

Lovis, C., Mayor, M., Pepe, F., et al. 2006, Nature, 441, 305

Mao, S., \& Paczynski, B. 1991, ApJ, 374, L37

Mayor, M. \& Queloz, D. 1995, Nature, 378, 355

Rattenbury, N. J., Bond, I. A., Skuljan, J., \& Yock, P. C. M. 2002, MNRAS, 335, 159

Rhie, S. H., Bennett, D. P., Becker, A. C., et al. 2000, ApJ, 533, 378

Rivera, E. J., Lissauer, J. J., Butler, R. P., et al. 2005, BAAS, 37, 1487

Snodgrass, C., Horne, K., \& Tsapras, Y. 2004, MNRAS, 351, 967

Tsapras, Y., Horne, K., Kane, S., \& Carson, R. 2003, MNRAS, 343, 1131

Udalski, A., Jaroszyński, M., Paczyński, B., et al. 2005, ApJ, 628, L109

Udry, S., Bonfils, X., Delfosse, X., et al. 2007, A\&A, 469, L43

Vermaak, P. 2000, MNRAS, 319, 1011

Wambsganss, J. 1997, MNRAS, 284, 172

Yoo, J., DePoy, D. L., Gal-Yam, A., et al. 2004, ApJ, 616, 1204 